Document downloaded from:

http://hdl.handle.net/10251/152273

This paper must be cited as:

Sanchez Fandiño, JA.; Muñoz Muñoz, P.; Doménech Gómez, JD.; Capmany Francoy, J. (2017). A monolithic integrated photonic microwave filter. Nature Photonics. 11(2):124-129. https://doi.org/10.1038/NPHOTON.2016.233

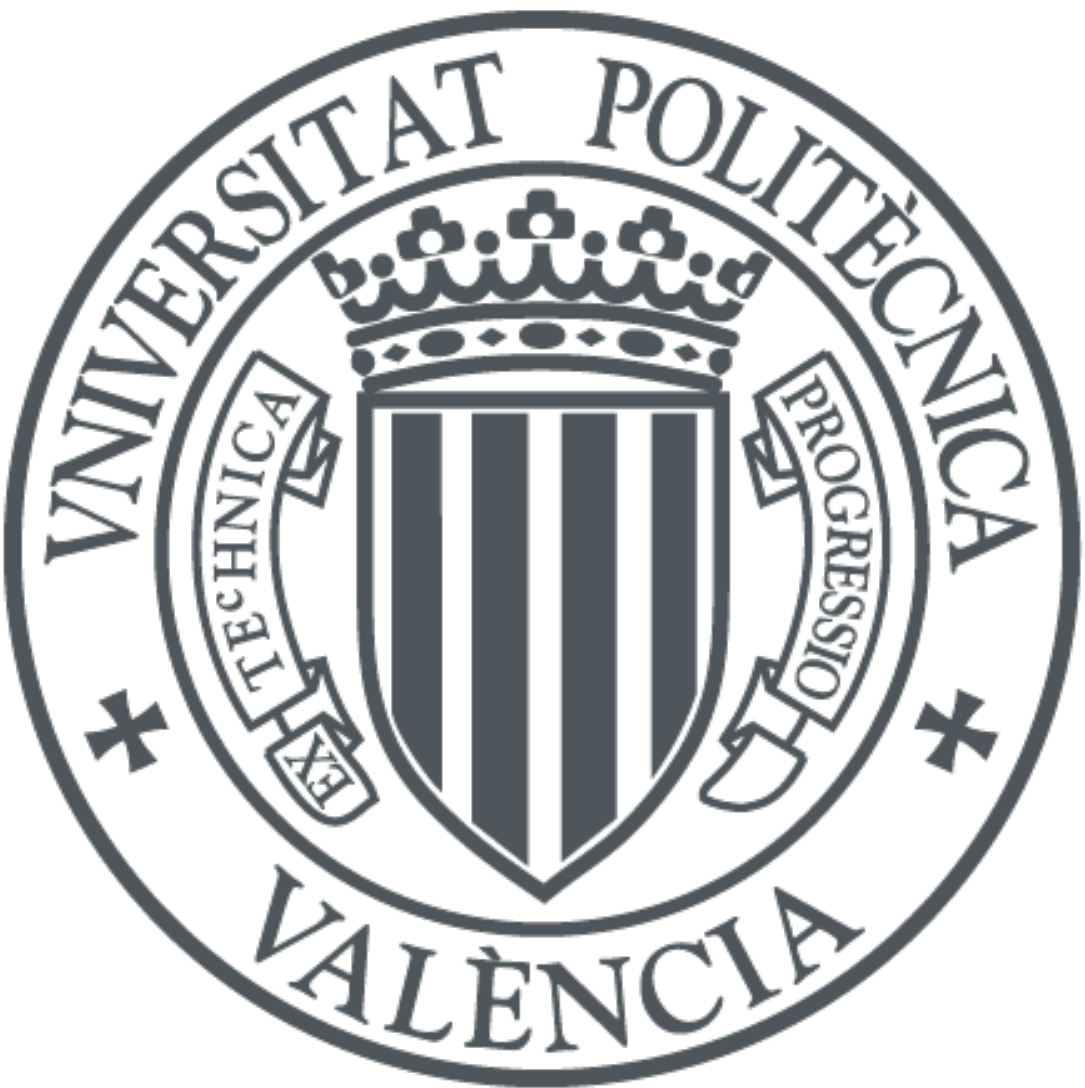

The final publication is available at

https://doi.org/10.1038/NPHOTON.2016.233

Copyright Nature Publishing Group

Additional Information 


\title{
A Monolithic Integrated Microwave Photonics Filter
}

\author{
Javier S. Fandiño${ }^{1}$, Pascual Muñoz ${ }^{1,2}$, David Doménech ${ }^{2}$, and José Capmany ${ }^{1}$ \\ 1iTEAM Research Institute, Universitat Politècnica de València, C/ Camino de Vera s/n, \\ Valencia 46022, Spain. e-mail: jcapmany@iteam.upv.es \\ ${ }^{2}$ VLC Photonics S.L., C/ Camino de Vera s/n, Valencia 46022, Spain. e-mail: \\ david.domenech@vlcphotonics.com
}

December 22, 2016

\begin{abstract}
Meeting the ever increasing demand for transmission capacity in wireless networks will require evolving towards higher regions in the radiofrequency spectrum, reducing cell sizes as well as resorting to more compact, agile and power efficient equipment at the base stations, capable of smoothly interfacing the radio and fiber segments. Photonic chips with fully functional microwave photonic systems are promising candidates to achieve these targets. Over the last years, many integrated microwave photonic chips have been reported in different technologies. However, and to the best of our knowledge, none of them have fully integrated all the required active and passive components. Here, we report the first ever demonstration of a microwave photonics tunable filter completely integrated in an Indium Phosphide chip and packaged. The chip implements a reconfigurable RF- photonic filter, it includes all the required elements, such as lasers, modulators and photodetectors, and its response can be tuned by means of control electric currents. This demonstration is a fundamental step towards the feasibility of compact and fully programmable integrated microwave photonic processors.
\end{abstract}

Emerging information technology scenarios, such as 5G mobile communications and Internet of Things (IoT), will require a flexible, scalable and future- proof solution capable for seamlessly interfacing the wireless and fiber segments of communication networks [1,2,3]. Microwave photonics (MWP) [4,5],the interdisciplin ary approach that combines radiofrequency and photonic systems, is the best positioned technology to achieve this target. A very relevant example is $5 \mathrm{G}$ wireless communications, which targets an extremely ambitious range of requirements including [6,7], a 1000-fold increase in capacity, connectivity for over 1 billion users, strict latency control, as well as network flexibility via agile software programming. These objectives call for a paradigm shift in the access network to incorporate smaller cells, exploit the millimeter wave regions of the radiofrequency spectrum and implement massive multiple-input multiple- output at the base stations (BTSs) [7]. The successful integration of the wireless and fiber segments thus relies on the possibility of implementing agile and reconfigurable MWP subsystems, featuring broadband operation, as well as low space, weight and power consumption metrics. The solution consists in resorting to integrated microwave photonics (IMWP) [8,9] chips allocated either in the BTS and/or the central office in combination with radio over fiber transmission in the fiber segment connecting them $[10,11]$. The two fundamental issues to be solved in IMWP are related respectively to technology and architecture. First, there is the need to identify the best material platform where to implement MWP chips. Second, whether it would be better to follow an application specific photonic integrated circuit (ASPIC) approach, where a specific architecture is employed to implement a specific functionality, or to resort to a generic programmable architecture. IMWP ASPICs with certain complexity have been reported to date mainly in four material platforms: indium phosphide (InP) [1214], Silicon-on-Insulator (SOI) [15-21], silicon nitride (Si3N4) [22-26] and chalcogenide glass [27,28]. Several functionalities have been demonstrated with a variable degree of photonic (20-60\%) integration, as shown in Table 1. A different approach is based on generic processors [29,30], where a common architecture implements different functionalities by suitable programming. A recent paper [31] reported the design of a programmable optical core inspired by the concept of electronic field programmable gate arrays. This approach is based on an optical core composed implemented by a $2 \mathrm{D}$ waveguide mesh where the connections between waveguides 
are controlled by means of tunable Mach-Zehnder interferometers (MZIs). Researchers fabricated a simplified version of the processor composed of two mesh cells, using a commercial Si3N4 waveguide technology known as TriPleX [32]. The reported processor featured a free spectral range of $14 \mathrm{GHz}$ and is fully programmable. A band-pass filter with a tunable centre frequency that spans two octaves (1.6-6 GHz) and a reconfigurable band shape (including a notch filter and a flat-top resonance) was demonstrated. A reconfigurable processor implementing signal integration, differentiation and Hilbert transformation has also been recently reported in InP technology [33]. To the best of our knowledge however, none of the above contributions has reported to date the integration of all the required active (sources, modulators and detectors) and passive (splitters, optical filters and waveguides) photonic components in a single chip, either monolithically or following a hybrid approach. Here we report, , the design, fabrication, packaging and experimental demonstration of the first monolithic IMPW filter that integrates all these elements in the same substrate. The chip implements a reconfigurable RF-photonic filter that employs a tunable distributed Bragg reflector laser (DBR); a singlesideband optical modulator; a tunable optical filter based on a ring assisted Mach Zehnder interferometer (RAMZI)[34]; and an on-chip optical detector. This demonstration constitutes a fundamental step forward in the implementation of a fully integrated MWP filter, and opens the path for compact and programmable MWP signal processors, where the RAMZI filter will be replaced by a 2D reconfigurable mesh and multiple functionalities will be implemented by suitable programming of the mesh interconnections.

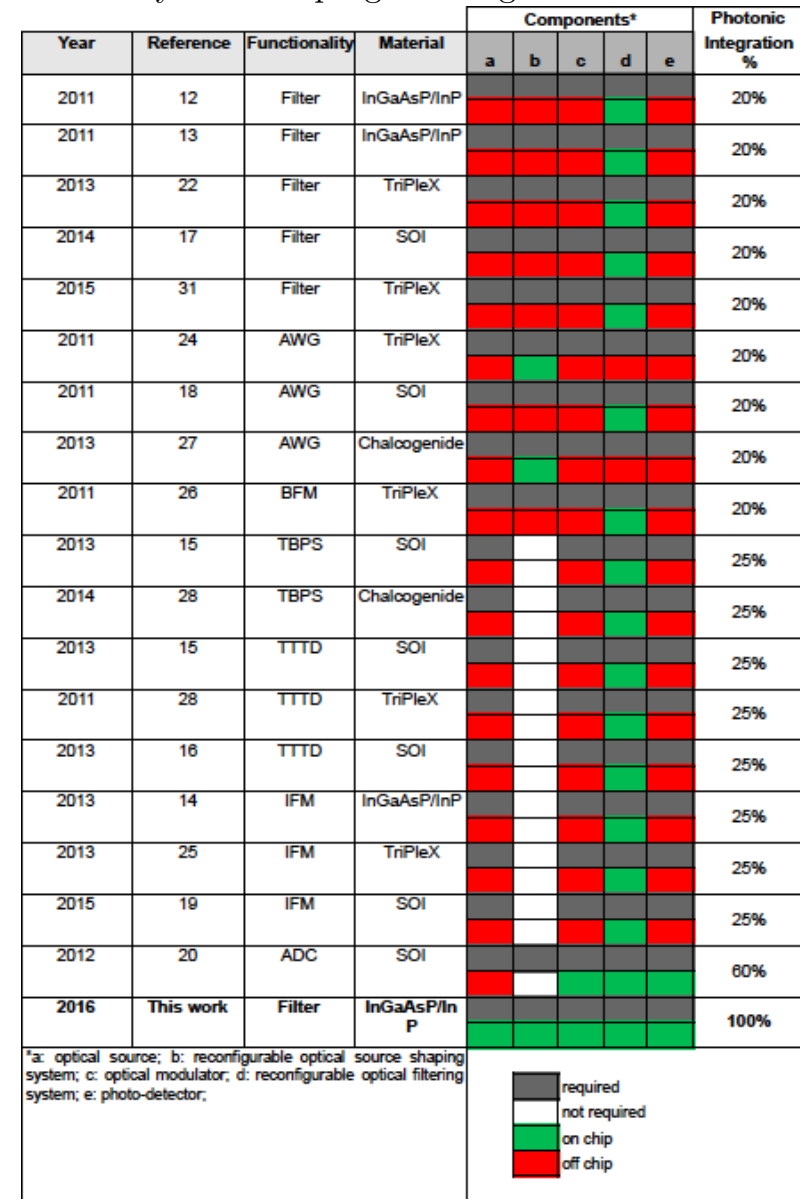

Table 1: Overview of reported IMWP chips. AWG: Arbitrary waveform generation. BFM: Beamforming. TBPS: Tunable broadband phase shift. TTTD: Tunable true-time delay. IFM: Instantaneous frequency measurement. ADC: Photonic analog-to-digital conversion. 


\section{Results}

\section{Basic concept}

Tunable MWP filtering is achieved in this photonic integrated circuit (PIC) by exploiting the well-known mapping between the optical and RF domains that happens when an optical single-sideband modulation (SSB) is sent through a tunable optical filter and is photodetected afterwards [35,36]. In brief, as shown in Fig. 1a, the output RF tone at a given modulation frequency is produced by the beating between the carrier and the optical SSB. As the modulation frequency is swept, the optical filter modifies the amplitude and phase of the sideband, while the carrier remains unchanged. This means that, after photodetection, the RF response of the MWP filter is just a scaled copy of the transfer function of the optical one, except for a constant amplitude and phase factor (see Operation principle in the Methods section). A MWP filter with a reconfigurable RF response can thus be obtained by combining a tunable laser, an optical SSB modulator, a photodetector and a tunable optical filter. The reconfigurability of the response lies here on both the tunable laser, whose central frequency can be changed to shift the whole RF transfer function, as well as on the tunable filter, which can change both the central frequency and the shape of the passband. Reconfigurable optical filters can be implemented for example with integrated optical lattice architectures [34,37]. Fig. 1b is a diagram of the designed InP PIC and its different building blocks (BBs). First, light coming out of a tunable distributed Bragg reflector laser (TL 1) is injected into a dual-drive modulator (MZM), which is made up of a symmetric Mach-Zehnder interferometer (MZI) with a couple of electro-optic phase shifters, one on each arm. By introducing two RF signals with a 90deg relative phase shift, and then setting the modulator at the quadrature bias point (90deg), an SSB modulation can be directly generated on-chip. This SSB signal is then sent through a an optical filter that can be reconfigured via thermo-optic heaters. The filter is based on the aforementioned RAMZI architecture, where ring resonators of the same perimeter are coupled to both branches of a symmetric Mach-Zehnder [38,39]. Because two $3 \mathrm{~dB}$ couplers are employed to split and recombine the signal in the interferometer arms, note that this filter architecture has two inputs and two outputs. One of the two available inputs is used to inject the SSB signal coming from the modulator into the filter. Afterwards, the modulated signal exists through one of the two outputs and is routed to an on-chip photodetector (PD 4), where the optical to electrical mapping finally takes place. The other free input of the RAMZI filter is routed with an auxiliary waveguide (called Input) to an optical spot-size converter located on one of the chip facets. The same is done for the other free output port, which is also routed with an auxiliary waveguide (called Output) to another spot- size converter on the same facet. The chip was manufactured and packaged using an InP generic integration technology on a multi-project wafer run [40] (see Fabrication and packaging in the Methods section for more details). Figures 1c-1e show a 3D representation of the PIC mask layout, a picture of a fabricated $6 \mathrm{x} 4 \mathrm{~mm} 2$ die, as well as a picture of one of the packaged chips, respectively. Note that the final chip layout includes more components that the ones described above, such as an extra tunable DBR laser laser, a phase modulator and a tunable 2x3 MZI with three DC photodiodes located at its output. These were employed when needed as auxiliary elements during individual characterization of some of the BBs of the MWP filter, like the tunable laser, the photodetector and the thermo-optic heaters. Experimental procedures and results for these elements are given as supplementary information (see Supplementary, Building block characterization section). Because of its importance, the RAMZI filter and the dual-drive MZM are described separately below.

\section{RAMZI filter}

Integrated optical filters are typically made up of a cascade (or lattice) of more basic, simpler optical elements, such as ring resonators and Mach-Zehnder interferometers [36]. Depending on the configuration and type of the employed basic unit cells, both finite impulse response (FIR) and infinite impulse response (IIR) optical filters can be implemented. In this case, a well-known architecture was chosen based on a symmetric MZI loaded with ring resonators, also known as RAMZI. A schematic diagram is shown in Fig. 2a. This architecture allows for the implementation of optimum IIR bandpass filters, such as the canonical Butterworth, Chebyshev and Elliptic approximations, while featuring a fewer number of optical elements as compared with other approaches [38]. In our case, the filter was designed as a fourth order Chebyshev type II filter, which ensures a flat passband and a sharper roll-off at the expense of ripples in the stopband. Note that the optical response is determined here by the coupling strength between the ring resonators and the MZI branches, as well as 
by the relative optical phase shifts of the rings and the MZI arms. The optical couplers that connect the rings with the arms of the Mach- Zehnder are fixed, and were implemented with custom $2 \times 2$ multimode interference couplers (MMIs) featuring a tapered body [41]. The optical phases can however be changed by means of thermo-optic heaters, which allow the tuning of the transfer function. Like any other optical lattice structure, it has a periodic frequency response, with a frequency periodicity commonly termed as Free Spectral Range (FSR). This is determined by the perimeter of the ring resonators and the group index of the waveguides, and it was designed to be $20 \mathrm{GHz}$. Ideal (no loss) magnitude and phase responses, as well as the simulated ones considering average propagation losses of $5.5 \mathrm{~dB} / \mathrm{cm}$, are shown in Fig. $2 \mathrm{~b}$ and 2c, respectively. In order to measure the tunable RAMZI filter, the auxiliary Input and Output waveguides (see Fig. 1b and 1c) were employed. These are coupled to a pair of single-mode fibers coming out of the metal package (Fig. 1e), which provide a mean for interfacing the RAMZI filter with external equipment. Two difficulties were nevertheless found during characterization of the filter. First, these fibers are not polarization maintaining. Thus, when light from an external source is injected into the chip, it is not generally possible to prevent the excitation of both quasi-TE and quasi-TM modes (dominant electric field component parallel/perpendicular to the chip surface, respectively) in the PIC waveguides to which those fibers are coupled. Since the chip was designed for TE polarization, care must be taken so that a random mix of polarizations is not excited simultaneously. To solve the aforementioned problem, a polarization alignment procedure was devised, which is described as supplementary information (see Supplementary, Polarization alignment procedure section). Second, different experiments showed that significant polarization rotation was taking place in the chip waveguides. These are also discussed with detail in the supplementary information (see Supplementary, RAMZI filter characterization section).

\section{Dual-drive MZM}

The purpose of the dual-drive MZM is to generate an SSB modulation. This requires the RF signals driving both arms to be 90deg out of phase, while the modulator should be biased at the quadrature point. The exact bias voltage that needs to be applied to the modulator for it to be at quadrature is generally unknown. A usual procedure is to connect the two RF inputs to a broadband 90deg RF hybrid, and then to slowly tune the bias point while observing the output modulation spectrum in an optical spectrum analyzer (OSA) for a fixed modulation frequency. A bias voltage is finally chosen for which the ratio between the powers of the lower and upper sidebands is maximum. This ratio is called SSB suppression $(\tau)$, and it is usually expressed in $\mathrm{dB}$.
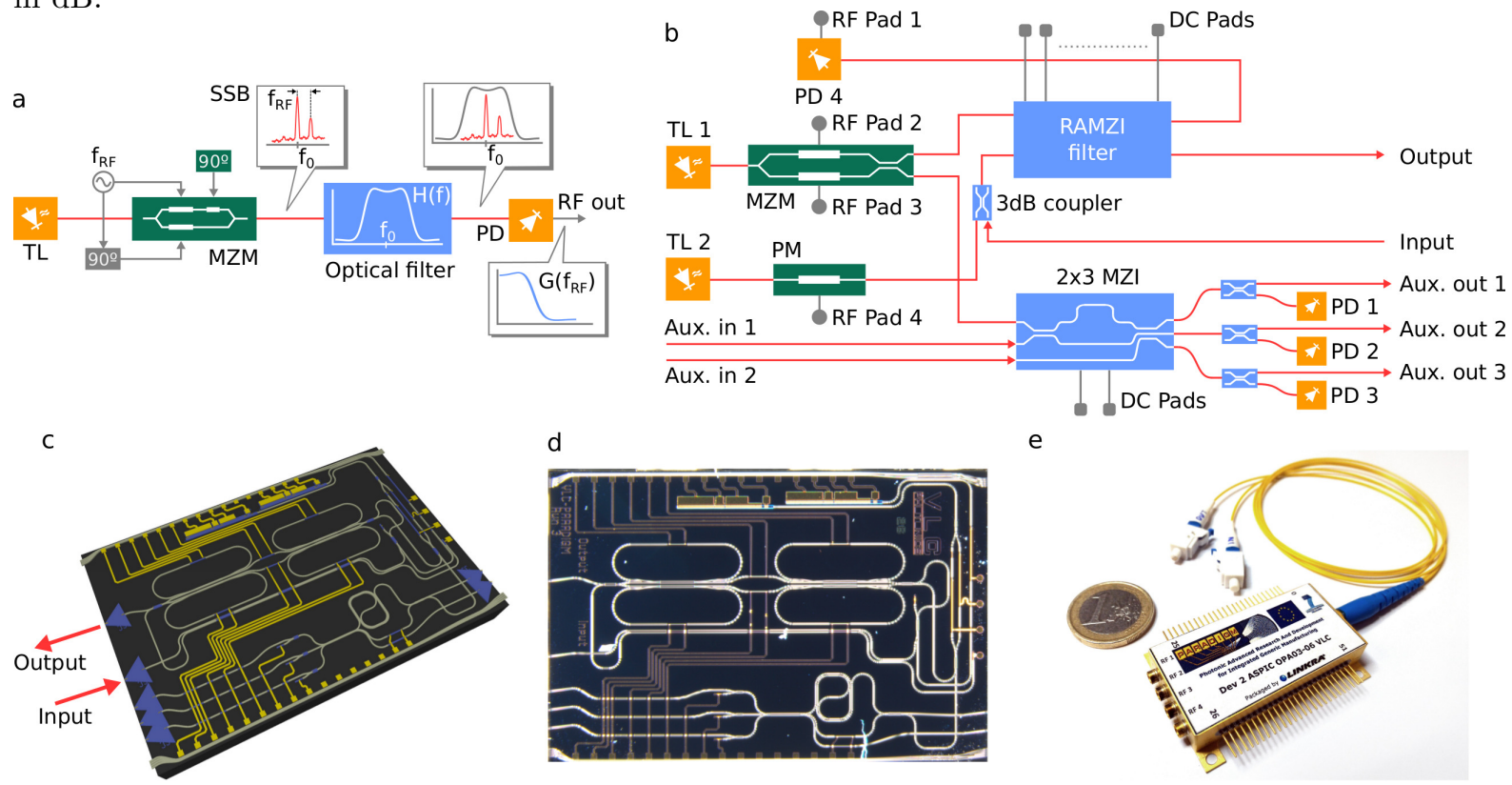

d

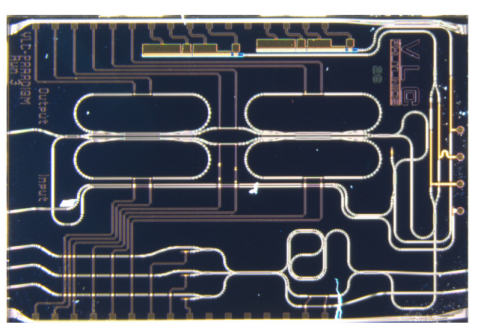

e

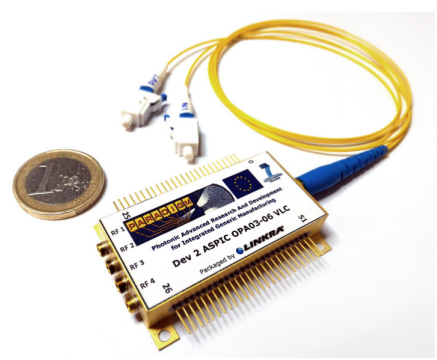

Fig. 1. Operation principle and schematic diagram of the integrated MWP filter. a, MWP filtering approach based on an optical SSB modulation and a tunable optical filter. b, Schematic of the InP chip, showing all the main building blocks. c, 3D representation of the chip layout. d, Picture of a fabricated 
die $(6 \times 4 \mathrm{~mm} 2)$. e, Packaged chip. TL: Tunable laser. MZM: Dual- drive Mach-Zehnder modulator. PD: Photodetector. PM: Phase modulator. MZI: Mach-Zehnder interferometer.

However, the layout of this PIC poses an extra difficulty: the optical spectrum measured at the output fiber is not that of the modulator itself, but that of the modulated signal after passing through the RAMZI filter. As a consequence, the measured ratio does not match with the real ratio at the output of the modulator. An alternative approach to solve this issue was devised (see Adjustment of the dual-drive MZM in the Methods section). The experimental setup for the MZM adjustment is shown in Fig. 2d, while the final results are shown in Figs. 2e-2f. Figure 2e shows the dependence of the median SSB suppression with bias voltage. As it can be seen, it reaches a maximum of around $14 \mathrm{~dB}$ at $-8 \mathrm{~V}$, which is the optimum bias point. This is better illustrated in the inset, where an overlap of all the measured modulation spectra (solid grey lines) is shown for this optimum voltage. They have been conveniently renormalized and shifted in wavelength, so that the fluctuations of the SSB suppression around the average value could be better appreciated. A black solid line represents the average of all these responses. Finally, Fig. $2 \mathrm{f}$ shows the experimental results for the same procedure, but this time keeping the optimum voltage $(-8 \mathrm{~V})$ and instead sweeping the modulation frequency with the same input RF power. As it can be seen, the achieved suppression is not constant with modulation frequency. For example, it goes below $5 \mathrm{~dB}$ for frequencies between 9 and $11 \mathrm{GHz}$, while it also shows a couple of dips around 7 and $8 \mathrm{GHz}$. This behaviour is attributed to poor RF performance of the modulator electrodes, due to impedance mismatch at the end of the RF line. At high frequencies a great part of the RF power is bouncing back through the transmission lines, creating standing wave patterns that are significantly affecting its broadband modulation behaviour, and introducing unwanted power and phase imbalances.

a
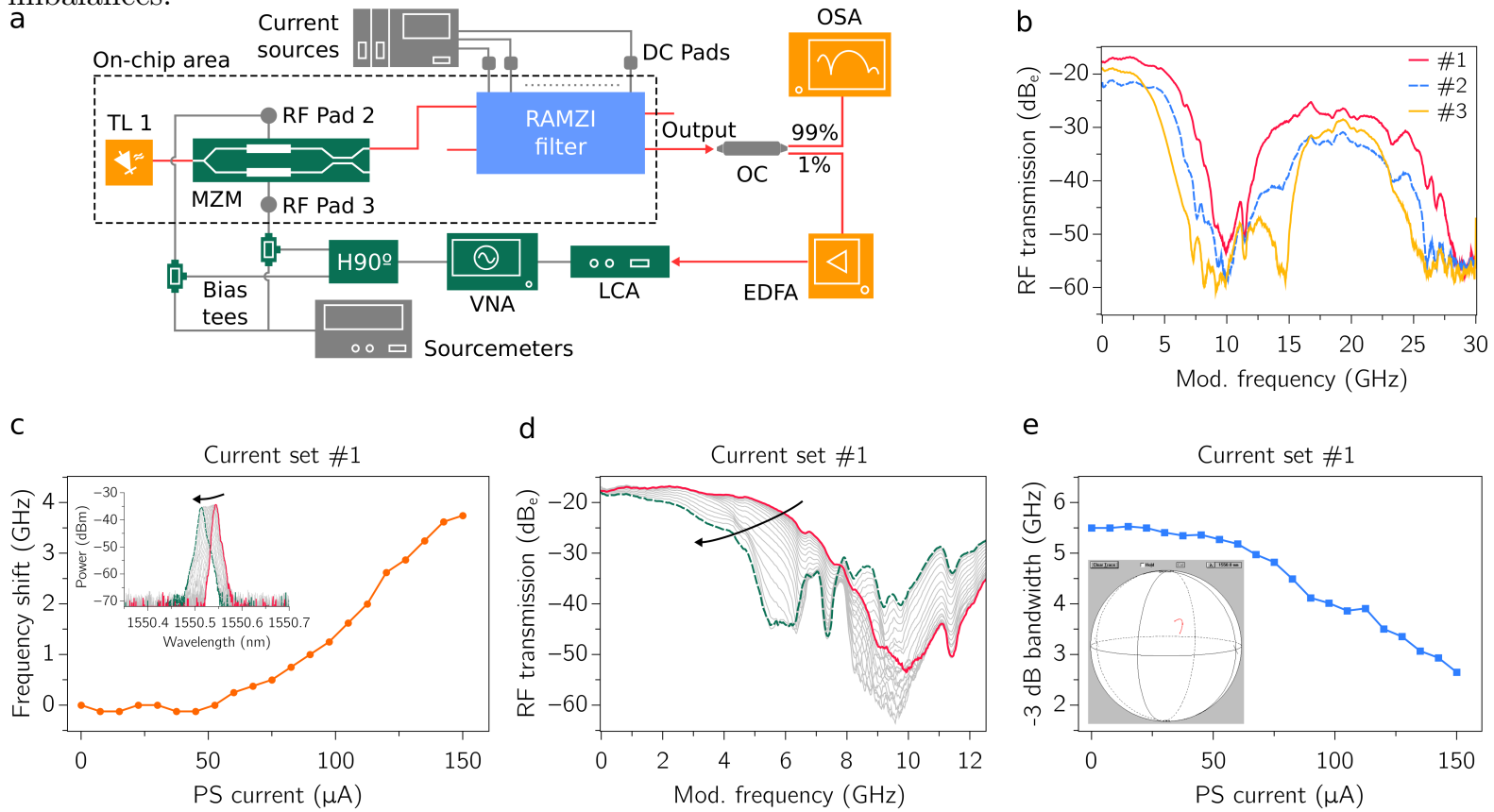

d

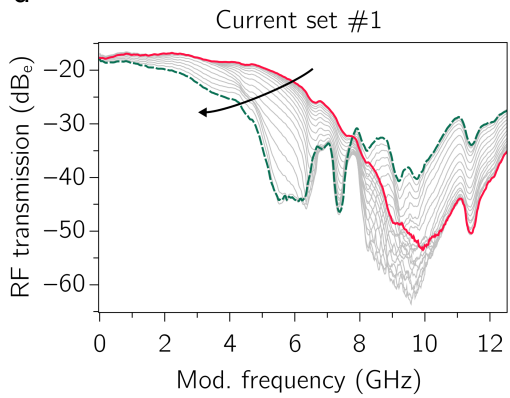

e

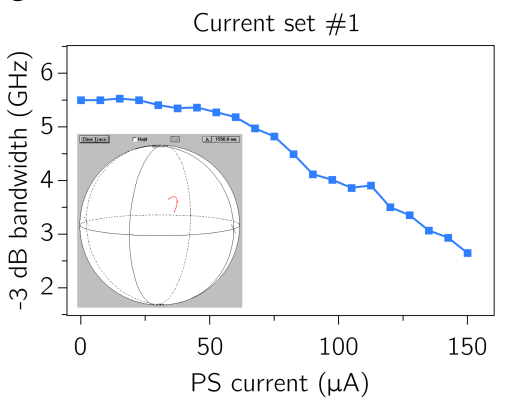

Fig. 2. RAMZI filter simulations and experimental adjustment of the dual- drive MZM. a, Diagram of the RAMZI filter. b, Ideal (solid grey line) magnitude response of the designed 4th order filter, and the simulated one (dashed magenta line) when considering propagation losses of $5.5 \mathrm{~dB} / \mathrm{cm}$. c, Idem for the phase response. d, Experimental setup for determining the optimum bias point of the dual-drive MZM. e, Dependence of the measured median sideband suppression with the applied bias voltage on one of the modulator arms, for a fixed modulation frequency of $8.5 \mathrm{GHz}$. The grey lines in the inset shows all the captured modulation spectra for the optimum bias point $(-8 \mathrm{~V})$, while the solid magenta line is the average of all these traces. f, Idem, when the bias is kept at the optimum point and the modulation frequency is swept between 6 and $12 \mathrm{GHz}$.

\section{Tunable MWP filter}

After the different BBs were characterized individually, two experiments were carried out to demonstrate the basic functionality of the MWP filter. The first one involved measuring its electro/optical (E/O) response. 
That is, modulating the on-chip dual-drive MZM and afterwards detecting the signal at the output of the RAMZI filter with an external photodetector connected to one of the fibers coming out of the PIC package. As discussed later in the text, a tunable low-pass MWP filter has been obtained with this approach, by first adjusting the transfer function of the RAMZI filter and then tuning the central wavelength of the on-chip laser. In the second experiment, a full electrical to electrical (E/E) measurement was performed. That is, an electrical RF signal was again injected into the on-chip dual-drive MZM, and the RF output at the integrated photodetector (PD 4) was then measured. However, the presence of significant electrical crosstalk was found to totally degrade the response of the MWP filter. This is a very important lesson to be learned when incorporating both driving and receiving RF electronics in the same chip and is discussed with more detail in the Electrical to Electrical (E/E) response of the MWP filter section of Supplementary. A schematic diagram of the E/O experiment is shown in Fig. 3a. First, the TL 1 is switched on, and a VNA and a 90deg RF hybrid are employed to modulate the dual-drive MZM. This is polarized at the optimum bias point (-8 V) to achieve the best SSB suppression. After the SSB modulation goes through the RAMZI filter, it is collected at the fiber, which is coupled to the auxiliary Output waveguide. Once the signal is outside the chip, it is split in two distinct paths by a $99 \%-1 \%$ optical coupler (OC). One one hand (1\%), it is injected into an OSA. On the other hand (99\%), it is amplified by an erbium-doped fiber amplifier (EDFA) and sent into a lightwave component analyzer (LCA, Agilent N4373C). Due to the polarization rotation issues described in the RAMZI filter characterization section (see Supplementary material), the polarization of the optical signal at the input of the RAMZI filter when coming from TL 1 is different to the one that results when an external laser source is coupled to the Input waveguide. Since the filter response is polarization dependent, the RAMZI filter will exhibit two different transfer functions in these two situations. This ultimately means that the response of the RAMZI filter as seen by the on-chip optical SSB modulation cannot be adjusted by measuring the filter with the external optical fibers, and must be optimized instead by directly looking at its E/O transfer function. During the experiment, the currents injected into the thermo-optic heaters were changed until a response resembling the target filter shape was obtained. We found three distinct sets of currents $(\# 1, \# 2$ and $\# 3)$ that yielded three different, although similar, responses. These are plotted in Fig. 3b. All of them feature a low-pass response with a $-3 \mathrm{~dB}$ bandwidth of $5.5,5$ and $3 \mathrm{GHz}$, respectively. Besides, note that they also exhibit a resonant peak near $20 \mathrm{GHz}$, which matches the target FSR of the filter.

a
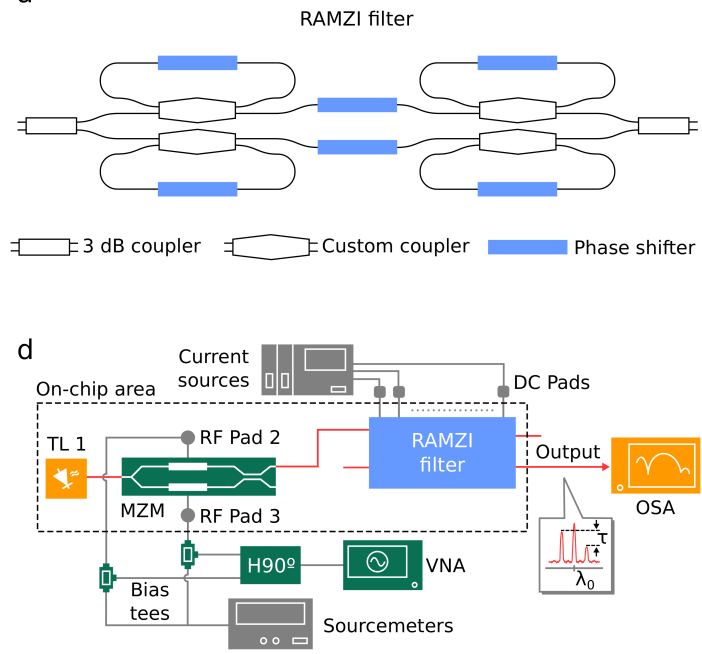

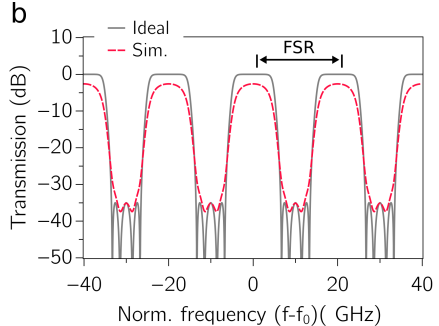

e

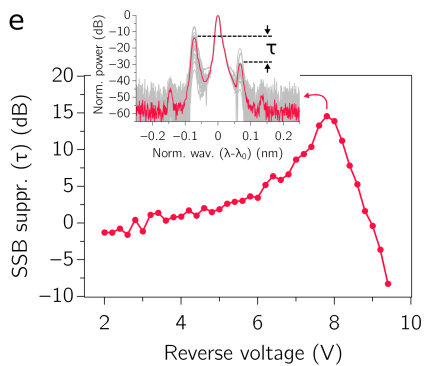

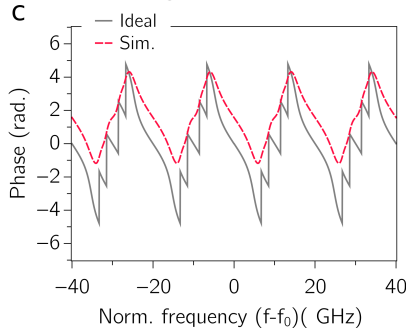

f

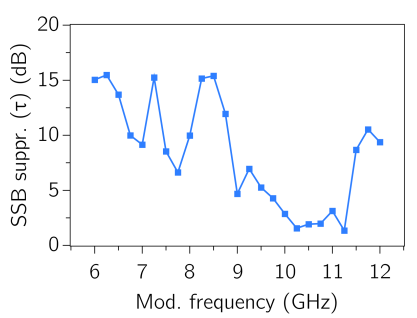

Fig. 3. Experimental results for the tunable MWP filter (E/O). a, Diagram of the experimental setup for the E/O measurement. b, Measured E/O responses for three different current sets in the thermo-optic heaters $(\# 1, \# 2, \# 3)$. c, Shift of TL 1 central frequency as a function of the current injected into its phase shifting section (PS), for the first current set (\#1). The inset shows the evolution of the unmodulated emission spectrum as measured with an OSA. d, Evolution of the measured E/O response as the frequency of TL 1 is shifted. e, Computed dependence of the $-3 \mathrm{~dB}$ bandwidth when current is injected into the PS. The inset shows the measured evolution of the polarization state on the Poincaré sphere at the output of the RAMZI filter.

To demonstrate the tunability of the MWP filter, several E/O responses for one of these current sets (\#1) 
were stored as different currents were applied to the PS shifting section of TL 1, which changed the center frequency of the laser. The changes in laser frequency were recorded by capturing the unmodulated output emission spectra in the OSA. Results are shown in Figs. 3c-3e. Figure 3c represents the positive shift in the central (optical) frequency of TL 1 (negative wavelength shift) as the current was increased in the PS section. It was shifted between 0 and $4 \mathrm{GHz}$ for when the current was increased from 0 to $150 \mu \mathrm{A}$. An overlapped representation of all the measured laser emission spectra is shown in the inset, when no RF power was driving the modulator. For a fixed optical frequency in TL 1, the different E/O responses obtained during the (RF) frequency sweep are shown in Fig. 3d. The solid magenta line represents the initial E/O response, when no current was injected into the PS, while the dashed, turquoise green line is the response that corresponds with the maximum injected current $(150 \mu \mathrm{A})$. The MWP filter exhibits tunable low-pass behaviour, where the width of the band is reduced by increasing the laser frequency. The measured $-3 \mathrm{~dB}$ bandwidth as a function of the tuning current is plotted in Fig. 3e. The $-3 \mathrm{~dB}$ bandwidth decreases from a maximum of $5.5 \mathrm{GHz}$ to a minimum of $2.5 \mathrm{GHz}$ (3 GHz range), which is similar to the increase in the frequency of the laser $(4 \mathrm{GHz})$ of Fig. 3c. The difference in these values, as well as the observed evolution of the RF filter shape for different PS currents, can be explained by both polarization rotation in the bends and the frequency- dependent SSB suppression of the dual-drive MZM. Polarization rotation is shown in the inset of Fig. 3e, where the measured polarization state of the unmodulated light coming out of the PIC during the frequency sweep is plotted in the Poincaré sphere. As the laser frequency increases, the polarization state of the signal inside the PIC changes, and so the effective transfer function of the RAMZI filter as seen by the SSB modulation.

\section{Discussion and summary}

In summary, we have reported on the design, fabrication and experimental demonstration of a monolithically integrated MWP filter in an InP PIC. The photonic packaged chip includes a tunable laser, photodetectors, a dual- drive modulator, as well as a RAMZI filter tunable via thermo-optic heaters. To the best of our knowledge, this is the first fully integrated MWP filter reported to date, and represents a significant step forward in the development of a generic integrated MWP processor, where the specific RAMZI optical filter will be replaced by a reconfigurable 2D mesh network. Integrated MWP filters are of special interest because of their superior tuning capabilities as compared to other RF technologies, and hold a significant potential as the next generation of mobile wireless standards move into higher frequency bands and require of wider bandwidths. During the course of the measurements, several detrimental effects, which will be fundamental in the process of implementing future integrated MWP processors, were identified and studied in more detail. In particular, a significant polarization rotation was observed in the deeply etched waveguides of the $\mathrm{InP}$ platform, which was later confirmed by means of simulations. This prevented the external measurement of the RAMZI filter, which could not be controlled and tuned to exactly match the original design target. Besides, severe RF crosstalk was observed when performing full electrical to electrical (E/E) measurements of the MWP filter. Nevertheless, the targeted tuning functionality was demonstrated using an electrical to optical (E/O) approach, where an auxiliary waveguide was employed to inject the SSB modulation at the output of the RAMZI filter into an external photodetector. A reconfigurable low-pass filter has been demonstrated this way, with a $-3 \mathrm{~dB}$ bandwidth tunable by $4 \mathrm{GHz}$ when changing the frequency of the on-chip tunable laser (TL 1). The hurdles found during the characterization stage are not intrinsic to the proposed technique, but rather due to fabrication imperfections and design issues, which can be well solved by a proper PIC redesign and careful dedicated manufacturing. Polarization rotation can be solved for example by either reducing the sidewall angle of the waveguides or using a much higher bending radius. Besides, the RF pads should be isolated and spaced as much as possible to prevent RF crosstalk. Finally, a proper termination of the dual-drive MZM arms with a right load would be beneficial to increase the performance and broadband behaviour of the SSB modulation. 


\section{Methods}

\section{Operation principle}

Assume that a laser with an average power P0 and center frequency f0 is injected into an SSB optical modulator, which is in turn being driven by an RF tone. The tone has the following form:

$$
V_{R F}(t)=A \cos \left(2 \pi f_{R F} t\right)
$$

where $\mathrm{A}$ is the $\mathrm{RF}$ voltage amplitude and $\mathrm{fRF}$ is the modulation frequency. The complex low-pass equivalent of the optical signal at the output of the modulator can be expressed, neglecting optical losses, as follows

$$
\tilde{E}_{S S B}(t)=\frac{\sqrt{P_{o}}}{2}\left[e^{j \pi V_{R F}(t)=A \cos \left(2 \pi f_{R F} t\right) / V_{\pi}}+j e^{j \pi V_{R F}(t)=A \sin \left(2 \pi f_{R F} t\right) / V_{\pi}}\right]
$$

Here, $\mathrm{V}$ stands for the $\pi$ voltage of each phase shifter, and we have also assumed that a 90deg RF hybrid is introducing a perfect $\pi / 2$ phase shift between the two RF signals that drive both arms of the SSB modulator. The modulator is also being biased at the quadrature point (90deg).

Usually, A is much lower than $\mathrm{V}$, so the small-angle approximation holds. That is, $\exp j x=\left(1-x^{2} / 2\right)+j x$ for $\mathrm{x} \ll 1$. For the sake of simplicity, second-order powers of the input signal are also neglected. Equation 2 can then be finally expressed as

$$
\tilde{E}_{S S B}(t)=\frac{\sqrt{P_{o}}}{2}\left[(1+j)+j \frac{\pi}{V_{\pi}} A e^{j 2 \pi f_{R F} t}\right]
$$

The Fourier transform of this waveform leads to

$$
\tilde{E}_{S S B}(f)=\frac{\sqrt{P_{o}}}{2}\left[(1+j) \delta(f)+j \frac{\pi}{V_{\pi}} A \delta\left(f-f_{R F}\right)\right]
$$

which corresponds with a single-sideband modulation. If this signal is introduced into an optical filter, then at the output we get

$$
\tilde{E}_{\text {out }}(f)=\frac{\sqrt{P_{o}}}{2}\left[(1+j) H(0) \delta(f)+j \frac{\pi}{V_{\pi}} A H\left(f_{R F}\right) \delta\left(f-f_{R F}\right)\right]
$$

where $\mathrm{H}(\mathrm{f})$ stands for the complex low-pass equivalent frequency response of the optical filter. That is, $\mathrm{H}(0)$ means that the filter is evaluated at the operating wavelength of the laser $\left(f_{0}\right.$, or $\lambda_{0}$ in the wavelength domain). The previous equation can be converted back to the time domain as

$$
\tilde{E}_{\text {out }}(t)=\frac{\sqrt{P_{o}}}{2}\left[(1+j) H(0)+j \frac{\pi}{V_{\pi}} A H\left(f_{R F}\right) e^{j 2 \pi f_{R F} t}\right]
$$

Finally, after detection, a time-varying photocurrent is obtained that is proportional to the modulus squared of [pic]. Neglecting the DC terms, and rewriting $\mathrm{H}(0)$ as $\kappa$, the $\mathrm{RF}$ component is given by

$$
i(t) \propto|\kappa| \mid H\left(f_{R F} \mid \cos \left(2 \pi f_{R F} t+\angle H\left(f_{R F}\right)+\angle \kappa\right)\right.
$$

Finally Equation 7 means that the RF response of the MWP filter $(\mathrm{G}(\mathrm{fRF}))$ is a scaled copy of the complex, low-pass equivalent of the optical filter transfer function $(\mathrm{H}(\mathrm{f}))$, multiplied by a complex constant $(\kappa)$. That is

$$
G\left(f_{R F}\right) \propto \kappa^{*} H\left(f_{R F}\right)
$$

As a consequence, a direct mapping between the optical and RF domains is obtained. 


\section{Device fabrication and packaging}

The designed PIC was manufactured within the european FP7 project PARADIGM (FP7-ICT-2009-5/257210). The PARADIGM initiative aimed at facilitating access to state-of-the-art InP foundries following a generic approach. That is, by providing external users with a set of predefined BBs that are not trivial to design, and then sharing the cost of PIC fabrication via multi-project wafer runs. In this case, the chip was manufactured in a non-insulating InP platform offered by the company Oclaro (United Kingdom). Deeply etched waveguides are made of an $\mathrm{n}$ - doped $\mathrm{InP}$ substrate over which an InGaAsP multi-quantum well structure is grown, forming the waveguide core. Afterwards, the core region is covered with a layer of p-doped InP. Finally, the whole structure is etched by about $3.6 \mu \mathrm{m}$ from the top of the p-doped region. The deep etch results in a high mode confinement, which allows for a tight bending radius of $150 \mu \mathrm{m}$. In this case, a waveguide width of $1.5 \mu \mathrm{m}$ was employed. According to the available data, it should experience propagation losses of about $5.5 \mathrm{~dB} / \mathrm{cm}$. Angled spot-size converters with both vertical and lateral tapers were also provided, featuring estimated coupling losses below $1 \mathrm{~dB}$. The PIC was packaged to facilitate its characterization. This service was offered by some partners of the PARADIGM consortium, and in this case was done by the company Linkra (Italy). The PIC layout was designed to meet the requirements imposed by the standard packaging process. This sets a maximum number of optical inputs/outputs, DC and RF pads; as well as it specifies a predefined location for all of them (see Figs. 1c-1e). A maximum of 48 DC pads can be employed, which are all located on the upper/lower facets of the die. Additionally, up to $4 \mathrm{RF}$ pads are available to the designer, all placed close to the right chip facet. Besides, up to two standard single-mode fibers can be coupled to on-chip spot-size converters, which are located on the left chip facet. Inside the metallic enclosure, the RF pads are wire-bonded to a ceramic substrate that routes the RF signals to external GPPO connectors (DC to $65 \mathrm{GHz}$ ). The DC pads are also wire-bonded and then connected to a set of equally spaced pins in the external metallic case. A thermistor and a Peltier cell are also included in the package to provide accurate temperature control.

\section{Adjustment of the dual-drive MZM}

The experimental setup employed to find the optimum bias point of the MZM is shown in Fig. 2d. It can be described as follows. A microwave vector network analyzer (VNA, Agilent PNA-X, N5245A, 10 MHz$50 \mathrm{GHz}$ ) first generates an RF tone with constant amplitude and frequency. This tone is injected into a $90^{\circ}$ RF hybrid (Marki microwave, QH0R714, 0.7-14.5 GHz), whose two outputs are then connected to a couple of broadband bias tees (SHF Communications, SHF-BT-45-D, $20 \mathrm{kHz}-45 \mathrm{GHz}$ ). In turn, they are used to drive the two ports of the dual-drive MZM (RF pads 2 and 3 in Fig. 1b). Now, for each reverse bias voltage in one of the MZM arms, we apply a different set of independent, uniformly distributed random currents in the thermo-optic heaters of the RAMZI filter, and finally capture the modulation spectrum exiting through the output fiber. The power ratio between the lower and upper sidebands $(\tau)$ is measured and stored for each current set. It is expected that the measured ratio will fluctuate up and down due to the influence of the RAMZI filter, which is changing for each set of random currents. However, if a sufficiently high number of modulation spectra are measured, then the median ratio extracted from these measurements will converge to the real value. That is, the real SSB suppression as it would be measured at the output of the modulator. In the experiment, a tone of $+5 \mathrm{dBm}$ and a frequency of $8.5 \mathrm{GHz}$ was first used to modulate the MZM. Besides, a constant reverse voltage of $-2 \mathrm{~V}$ was applied in one of the MZM arms, while the bias in the other arm was swept between 2 and $9.4 \mathrm{~V}$ in steps of $0.2 \mathrm{~V}$. For each bias value, we applied a set of 25 different, independent, uniformly distributed random currents in the 6 thermo-optic heaters of the RAMZI filter, and the output spectrum was captured in an OSA (Advantest Q8384).

\section{References}

1. Novak, D. et al., Radio-Over-Fiber Technologies for Emerging Wireless Systems. IEEE J. of Quantum Electron. 52, 1-11 (2016).

2. Waterhouse, R. \& Novak, D. Realizing 5G: Microwave Photonics for 5G Mobile Wireless Systems. IEEE Microw. Mag. 16, 84-92 (2015). 
3. Technology Focus on Microwave Photonics. Nature Photon. 5, 723 (2011).

4. Capmany, J. \& Novak, D. Microwave Photonics combines two worlds. Nature Photon. 1, 319-330 (2007).

5. Yao, J. Microwave Photonics. J. Lightw. Technol. 27, 314-335 (2009).

6. Andrews, J.G., Buzzi, S., Wan, C., Hanly, S.V., Lozano, A., Soong, A.C.K. \& Zhang, J.C. What Will 5G Be? IEEE J. Sel. Areas Commun. 32, 1065-1082 (2014).

7. Gosh, Al. et al. Millimeter-Wave Enhanced Local Area Systems: A High-Data- Rate Approach for Future Wireless Networks. IEEE J. Sel. Areas Commun. 32, 1152-1163 (2014).

8. Marpaung, D. et al. Integrated Microwave Photonics. Laser Photon. Rev. 7, 506-538 (2013).

9. Iezekiel, S., Burla, M., Klamkin, J., Marpaung, D. \& Capmany, J. RF Engineering Meets Optoelectronics: Progress in Integrated Microwave Photonics. IEEE Microw. Mag. 16, 28-45 (2015).

10. Mitchell, J.E. Integrated Wireless Backhaul Over Optical Access Networks. J. Lightw. Technol. 32, 3373-3382 (2014).

11. Liu, C., Wang, J., Cheng, L., Zhu, M. \& Chang, G.-K. Key. Key Microwave- Photonics Technologies for Next-Generation Cloud-Based Radio Access Networks. J. Lightw. Technol. 32, 3452-3460 (2014).

12. Norberg, E.J., Guzzon, R.S., Parker, J.S., Johansson, L.A. \& Coldren, L.A. Programmable Photonic Microwave Filters Monolithically Integrated in InP/InGaAsP. J. Lightw. Technol. 29, 1611-1619 (2011).

13. Guzzon, R., Norberg, E., Parker, J., Johansson, L. \& Coldren, L. Integrated InP-InGaAsP tunable coupled ring optical bandpass filters with zero insertion loss. Opt. Express 19, 7816-7826 (2011).

14. Fandino, J.S. \& Munoz, P. Photonics-based microwave frequency measurement using a double-sideband suppressed-carrier modulation and an InP integrated ring-assisted Mach-Zehnder interferometer filter. Opt. Lett. 38, 4316-4319 (2013).

15. Burla, M., Cortes, L.R., Li, M., Wang, X., Chrostwoski, L. \& Azaña, J. On- chip ultra-wideband microwave photonic phase shifter and true time delay line based on a single phase-shifted waveguide Bragg grating. in IEEE International Topical Meeting on Microwave Photonics, 92-95 (2013).

16. Shi, W., Veerasubramanian, V., Patel, D. \& Plant, D. Tunable nanophotonic delay lines using linearly chirped contradirectioinal couplers with uniform Bragg gratings. Opt. Lett. 39, 701-703 (2014).

17. Guan, B. et al. CMOS Compatible Reconfigurable Silicon Photonic Lattice Filters Using Cascaded Unit Cells for RF-Photonic Processing. IEEE J. Sel. Topics Quantum Electron. 20, 359-368 (2014).

18. Khan, M.H., Shen, H., Xuan, Y., Zhao, L., Xiao, S., Leaird, D.E., Weiner, M.A. \& Qi, M. Ultrabroadbandwidth arbitrary radiofrequency waveform generation with a silicon photonic chip-based spectral shaper. Nature Photon. 4, 117-122 (2010).

19. Pagani, M. et al. Instantaneous frequency measurement system using four- wave mixing in an ultracompact long silicon waveguide. in European Conference on Optical Communication (ECOC), 1-3 (2015).

20. Khilo, A.. et al. Photonic ADC: overcoming the bottleneck of electronic jitter. Opt. Express 20, 4454-4469 (2012).

21. Wang, J. et al. Reconfigurable radio-frequency arbitrary waveforms synthesized in a silicon photonic chip. Nat. Commun. 6, 5957 (2015).

22. Marpaung, D., Morrison, B., Pant, R., Roeloffzen, C., Leinse, A., Hoekman, M., Heideman, R. \& Eggleton, B.J. Si3N4 ring resonator-based microwave photonic notch filter with an ultrahigh peak rejection. Opt. Express 21, 23286-23294 (2013). 
23. Zhuang, L., Taddei, C., Hoekman, M., Leinse, A., Heideman, R., van Dijk, P. \& Roeloffzen, C. Ring resonator-based on-chip modulation transformer for high-performance phase-modulated microwave photonic links. Opt. Express 21, 25999-26013 (2013).

24. Marpaung, D., Chevalier, L., Burla, M. \& Roeloffzen, C. Impulse radio ultrawideband pulse shaper based on a programmable photonic chip frequency discriminator. Opt. Express 19, 24838-24848 (2011).

25. Marpaung, D. On-Chip Photonic-Assisted Instantaneous Microwave Frequency Measurement System. IEEE Photon. Technol. Lett. 25, 837-840 (2013).

26. Burla, M., Marpaung, D., Zhuang, L., Roeloffzen, C., Khan, M.R., Leinse, A., Hoekman, M. \& Heideman, R. On-chip CMOS compatible reconfigurable optical delay line with separate carrier tuning for microwave photonic signal processing. Opt. Express 19, 21475-21484 (2011).

27. Tan, K. et al. Photonic-chip-based all-optical ultra-wideband pulse generation via XPM and birefringence in a chalcogenide waveguide. Opt. Express 21, 2003-2011 (2013).

28. Pagani, M. et al. Tunable wideband microwave photonic phase shifter using on-chip stimulated Brillouin scattering. Opt. Express 22, 28810-28818 (2014).

29. Perez, D., Gasulla, I. \& Capmany, J. Software-defined reconfigurable microwave photonics processor. Opt. Express 23, 14640-14654 (2015).

30. Capmany, J., Gasulla, I. \& Pérez, D. Microwave photonics: The programmable processor. Nature Photon. 10, 6-8 (2016).

31. Zhuang, L., Roeloffzen, C.G.H., Hoekman, M., Boller, K.-J. \& Lowery, A.J. Programmable photonic signal processor chip for radiofrequency applications. Optica 2, 854-859 (2015).

32. Roeloffzen, C.G. et al. Silicon nitride microwave photonic circuits. Opt. Express 21, 22937-22961 (2013).

33. Liu, W. et al. A fully reconfigurable photonic integrated signal processor. Nature Photon. 10, 190-195 (2016).

34. Madsen, C.K. \& Zhao, J. H., Optical Filter Design and Analysis: A Signal Processing Approach. Wiley (1999).

35. Roman, J., Frankel, M.Y. \& Esman, R.D. Spectral characterization of fiber a gratings with high resolution. Opt. Lett. 23, 939-941 (1998).

36. Hernandez, R., Loayssa, A. \& Benito, D. Optical vector network analysis based on single-sideband modulation. Opt. Eng. 43, 2418-2421 (2004).

37. Jinguji, K. \& Oguma, M. Optical Half-Band Filters. J. Lightw. Technol. 18, 252-259 ( 2000).

38. Madsen. C.K. Efficient Architectures for Exactly Realizing Optical Filters with Optimum Bandpass Designs. IEEE Photon. Technol. Lett. 10, 1136-1138 (1998).

39. Madsen, C.K. General IIR Optical Filter Design for WDM Applications Using All-Pass Filters. J. Lightw. Technol. 18, 860-868 (2000).

40. M.K. Smit et al., "An introduction to InP-based generic integration technology," Semiconductor Science and Technology, vol. 29, no 8, p. 083001 (2014).

41. Besse, P.A., Gini, E., Bachmann, M. \& Melchior, H. New 2x2 and 1x3 Multimode Interference Couplers with Free Selection of Power Splitting Ratios. J. Lightw. Technol. 14, 2286-2293 (1996).

42. Halir, R., Vivien, L., Le Roux, X., Xu, D.-X. \& Cheben, P. Direct and Sensitive Phase Readout for Integrated Waveguide Sensors. IEEE Photon. J. 5, 6800906 (2013). 
43. van Dam, C. et al. Novel Compact Polarization Converters Based on Ultra Short Bends. IEEE Photon. Technol. Lett. 8, 1346-1348 (1996).

44. Morichetti, F., Melloni, A. \& Martinelli, M. Effects of Polarization Rotation in Optical Ring-ResonatorBased Devices. J. Lightw. Technol. 24, 573-585 (2006).

Acknowledgments. The authors acknowledge financial support by the Spanish CDTI NEOTEC start-up programme, the European Commission FP7-PARADIGM project, the Generalitat Valenciana PROMETEO 2013/012 research excellency award, the Spanish MINECO project TEC2013-42332-P, acronym PIF4ESP, project FEDER UPVOV 10-3E-492 and project FEDER UPVOV 08-3E-008. J.S. Fandiño acknowledges financial support through FPU grant AP2010-1595.

Author contributions. J.S.F. and D.D. designed the chip. J.S.F. conceived the experiments and performed the measurements. J.S.F., P.M. and J.C. analyzed the data and wrote the paper. P.M and J.C managed the project.

Additional information. The authors declare no competing financial interests. Reprints and permission information is available online at http://npg.nature.com/reprintsandpermissions/. Correspondence and requests for materials should be addressed to J.C. 